\title{
Exponential Estimates for Solutions of Linear Systems With Distributed Delay
}

\author{
Irina V. Medvedeva \\ St. Petersburg State University \\ 7/9 Universitetskaya nab., St. Petersburg, 199034, Russia \\ Email: medv.irina@gmail.com, i.medvedeva@spbu.ru
}

\begin{abstract}
In this paper, for a class of linear time-invariant systems with distributed delay, the robust stability conditions are obtained, and the exponential estimates for the solutions are constructed. The results are derived within the framework of the Lyapunov-Krasovskii functionals with a given derivative. The functional, that does not admit a quadratic lower bound and therefore is considered to be inappropriate for the robustness analysis, is used.
\end{abstract}

\section{INTRODUCTION}

The present paper focuses on two particular problems, namely the robustness analysis and the construction of the exponential estimates for solutions, for linear time-invariant differential systems with distributed delay. These problems are treated on the basis of the Lyapunov-Krasovskii functionals approach that goes back to the works [5] and [11] and was well developed in the last few decades, see the recent book [7]. In [4], the quadratic functionals with a given time-derivative (chosen first as a quadratic form of the vector $x(t)$ by analogy to the classical Lyapunov functions) were presented. Further the structure of the derivative was refined: it became the functional, and in this way the so-called complete-type functionals were obtained, see [6] for systems with distributed time delay. The complete-type functionals were shown to be effective in solving the problems we address in this paper: for systems with concentrated delays, they were applied to obtention of the robustness bounds (see [7]) and to construction of the exponential estimates (see [8]). The results were generalized to the distributed delay case in [12] and [13]. The main point, allowing the functionals to be useful, is that they admit the quadratic lower bounds, see [12] for the distributed delay case.

In this paper, we present a different approach to deal with the problems in question. It uses the functionals referred in [4]. Although there are no quadratic lower bounds for these functionals, recently it was shown that such bounds exist on some special set of functions (see Theorem 1) [9]. The latter result allows to apply the functionals to the robustness analysis. The examples show that in some cases the robustness bounds obtained within our approach are significantly less conservative than those obtained with the help of the completetype functionals. Note that methodology of [12] do not work with the functionals we use. The paper presents an extension of the work [10] to the distributed delay case.
Notation: Throughout the paper, we use the euclidian norm for vectors; notation $I$ means the identity matrix; $\lambda_{\min }(W)$ is the minimal eigenvalue of the symmetric matrix $W$.

\section{PRELIMINARIES}

We consider a linear time-invariant system with distributed time-delay

$$
\begin{aligned}
\dot{x}(t)=A_{0} x(t) & +A_{1} x(t-h) \\
& +\int_{-h}^{0} G(\theta) x(t+\theta) d \theta, \quad t \geqslant 0 .
\end{aligned}
$$

Here $A_{0}, A_{1} \in \mathbb{R}^{n \times n}$ are the constant matrices, $h>0$ is the constant delay, and $G(\theta)$ is continuous matrix-valued function. Suppose that the initial function $\varphi(\theta), \theta \in[-h, 0]$, is piecewise continuous, and define the uniform norm

$$
\|\varphi\|_{h}=\sup _{\theta \in[-h, 0]}\|\varphi(\theta)\|
$$

on the space of the piecewise continuous functions. Let $x(t, \varphi)$ stand for the solution of system (1) with the initial function $\varphi$, and

$$
x_{t}(\varphi): \theta \rightarrow x(t+\theta, \varphi), \theta \in[-h, 0],
$$

be the segment of solution.

Following the traditional concept (see [1] and [7]), we say that system (1) is exponentially stable, if there exist $\gamma \geqslant 1$ and $\sigma>0$ such that

$$
\|x(t, \varphi)\| \leqslant \gamma e^{-\sigma t}\|\varphi\|_{h}, \quad t \geqslant 0,
$$

for every solution of system (1). As the aim of the present paper is to analyze the robustness and to construct the exponential estimates for solutions, throughout the paper we suppose that system (1) is exponentially stable.

In this work, we use the functional whose time-derivative along the solutions of system (1), $d v\left(x_{t}\right) / d t$, coincides with the quadratic form $-x^{T}(t) W x(t)$, where $W$ is a given positive definite matrix. According to [4] and [7], this functional is of the form

$$
\begin{aligned}
v(\varphi) & =\varphi^{T}(0) U(0) \varphi(0)+2 \varphi^{T}(0) \int_{-h}^{0} U(-\theta-h) A_{1} \varphi(\theta) d \theta \\
& +\int_{-h}^{0} \int_{-h}^{0} \varphi^{T}\left(\theta_{1}\right) A_{1}^{T} U\left(\theta_{1}-\theta_{2}\right) A_{1} \varphi\left(\theta_{2}\right) d \theta_{1} d \theta_{2}
\end{aligned}
$$




$$
\begin{gathered}
+2 \varphi^{T}(0) \int_{-h}^{0}\left(\int_{-h}^{\theta} U(\xi-\theta) G(\xi) d \xi\right) \varphi(\theta) d \theta \\
+2 \int_{-h}^{0} \int_{-h}^{0} \varphi^{T}\left(\theta_{1}\right) A_{1}^{T}\left(\int_{-h}^{\theta_{2}} U\left(h+\theta_{1}-\theta_{2}+\xi\right) G(\xi) d \xi\right) \\
\times \varphi\left(\theta_{2}\right) d \theta_{1} d \theta_{2}+\int_{-h}^{0} \int_{-h}^{0} \varphi^{T}\left(\theta_{1}\right)\left(\int_{-h}^{\theta_{1}} \int_{-h}^{\theta_{2}} G^{T}\left(\xi_{1}\right)\right. \\
\left.\times U\left(\theta_{1}-\theta_{2}-\xi_{1}+\xi_{2}\right) G\left(\xi_{2}\right) d \xi_{1} d \xi_{2}\right) \varphi\left(\theta_{2}\right) d \theta_{1} d \theta_{2},
\end{gathered}
$$

where $U(\tau)$ is the Lyapunov matrix associated with $W$, see [6] or [7]. If the system is exponentially stable, the Lyapunov matrix is continuous, hence there exists

$$
M=\max _{\tau \in[0, h]}\|U(\tau)\| .
$$

Moreover, as a direct consequence of the results of works [3] and [2], it can be shown that

$$
M=\|U(0)\| .
$$

The following exponential stability criterion is the basis of the present paper.

Theorem 1. [9] Given a positive definite matrix $W$, system (1) is exponentially stable, if and only if functional (3) admits the following lower bound

$$
v(\varphi) \geqslant \mu\|\varphi(0)\|^{2}, \quad \varphi \in S,
$$

where $\mu>0$ and $S$ is the set of the piecewise continuous functions such that $\|\varphi(\theta)\| \leqslant\|\varphi(0)\|, \theta \in[-h, 0]$.

\section{RoBUSTNESS BoUndS}

Let system (1) be exponentially stable. Together with system (1) consider the following perturbed system

$$
\begin{aligned}
\dot{y}(t) & =\left(A_{0}+\Delta_{0}\right) y(t)+\left(A_{1}+\Delta_{1}\right) y(t-h) \\
& +\int_{-h}^{0}\left(G(\theta)+\Delta_{2}(\theta)\right) y(t+\theta) d \theta,
\end{aligned}
$$

where the perturbation matrices $\Delta_{0}, \Delta_{1}$, and $\Delta_{2}(\theta)$ are supposed to satisfy the inequalities

$$
\left\|\Delta_{0}\right\| \leqslant \rho_{0},\left\|\Delta_{1}\right\| \leqslant \rho_{1},\left\|\Delta_{2}(\theta)\right\| \leqslant \rho_{2}, \theta \in[-h, 0] .
$$

The aim of this section is to estimate the values $\rho_{j}, j=0,1,2$, for which system (4) remains exponentially stable.

The idea of the technique is borrowed from [7] and is as follows: to analyze the stability of system (4), we use the functional $v(\varphi)$ corresponding to the original system (1). The calculations show that the time-derivative of functional (3) along the solutions of the perturbed system has the form [12]

$$
\frac{d v\left(y_{t}\right)}{d t}=-y^{T}(t) W y(t)+l\left(y_{t}\right)
$$

where

$$
\begin{aligned}
l\left(y_{t}\right) & =2\left(\Delta_{0} y(t)+\Delta_{1} y(t-h)+\int_{-h}^{0} \Delta_{2}(\xi) y(t+\xi) d \xi\right)^{T} \\
& \times\left[U(0) y(t)+\int_{-h}^{0} U(-\theta-h) A_{1} y(t+\theta) d \theta\right. \\
& \left.+\int_{-h}^{0}\left(\int_{-h}^{\theta} U(\xi-\theta) G(\xi) d \xi\right) y(t+\theta) d \theta\right] .
\end{aligned}
$$

The functional $l\left(y_{t}\right)$ admits the following upper bound:

$$
\begin{aligned}
l\left(y_{t}\right) & \leqslant M\left(\left((\alpha+1) \rho_{0}+\rho_{1}+h \rho_{2}\right)\|y(t)\|^{2}\right. \\
& +\alpha \rho_{1}\|y(t-h)\|^{2} \\
& \left.+\left(\alpha \rho_{2}+L\left(\rho_{0}+\rho_{1}+h \rho_{2}\right)\right) \int_{-h}^{0}\|y(t+\theta)\|^{2} d \theta\right),
\end{aligned}
$$

where

$$
\alpha=1+L h, \quad L=\left\|A_{1}\right\|+g h, \quad g=\max _{\theta \in[-h, 0]}\|G(\theta)\| .
$$

Hence, by direct integration, we obtain

$$
\begin{gathered}
\int_{0}^{t} l\left(y_{s}\right) d s \leqslant 2 \alpha M\left(\rho_{0}+\rho_{1}+h \rho_{2}\right) \int_{0}^{t}\|y(s)\|^{2} d s \\
+M\left(\alpha\left(\rho_{1}+h \rho_{2}\right)+\operatorname{Lh}\left(\rho_{0}+\rho_{1}+h \rho_{2}\right)\right) \int_{-h}^{0}\|\varphi(s)\|^{2} d s .
\end{gathered}
$$

The second summand in the latter estimate is always bounded. So it can be shown that, although time-derivative (5) can not be done negative definite by the choice of $\rho_{j}$, the positiveness of the expression

$$
\lambda_{\min }(W)-2 \alpha M\left(\rho_{0}+\rho_{1}+h \rho_{2}\right)
$$

is sufficient for the exponential stability of system (4):

Theorem 2. If system (1) is exponentially stable and

$$
\begin{gathered}
\rho_{0}+\rho_{1}+h \rho_{2}<\frac{\lambda_{\min }(W)}{2 \alpha M}, \text { where } \\
\alpha=1+\left\|A_{1}\right\| h+g h^{2}, g=\max _{\theta \in[-h, 0]}\|G(\theta)\|, \quad M=\|U(0)\|,
\end{gathered}
$$

then the perturbed system (4) remains exponentially stable.

\section{EXPONENTIAL Estimates}

The aim of this section is to find the estimations for the values $\gamma$ and $\sigma$ from the definition of the exponential stability on the basis of Theorems 1 and 2 .

Consider system (1), and make the change of variable according to the formula $y(t)=e^{\sigma t} x(t)$ for some $\sigma>0$, then we obtain

$$
\begin{aligned}
\dot{y}(t)=\left(A_{0}+\sigma I\right) y(t) & +e^{\sigma h} A_{1} y(t-h) \\
& +\int_{-h}^{0} e^{-\sigma \theta} G(\theta) y(t+\theta) d \theta .
\end{aligned}
$$

The application of Theorem 2 to system (6) leads to the following result. 
Theorem 3. If system (1) is exponentially stable and

$$
\sigma+L\left(e^{\sigma h}-1\right)<\frac{\lambda_{\min }(W)}{2 \alpha M},
$$

where $L=\left\|A_{1}\right\|+g h$, and $\alpha, g, M$ are the same as in Theorem 2, then system (6) is also exponentially stable.

Inequality (7) provides a lower bound for the value of $\sigma$ from the definition (2). Moreover, it can be shown that making a few iterations we can construct a sequence of such bounds converging to the exact value of decay rate of system (1), see [10] for more details in case of concentrated delays.

Now we are going to provide the estimation for the $\gamma$-factor from the inequality (2), and we will do it in two ways. In the first one, we set some $\sigma$ satisfying (7) and use functional (3) corresponding to system (6), let us denote it by $v_{\sigma}(\varphi)$. In the second one, we use functional (3) corresponding to the original system (1).

It is known that functional (3) admits the following upper bound [7]

$$
v(\varphi) \leqslant \eta\|\varphi\|_{h}^{2}, \text { where } \eta=M \alpha^{2},
$$

$M$ and $\alpha$ are defined in Theorem 2. Hence, for the functional $v_{\sigma}(\varphi)$ we can write

$$
\begin{gathered}
v_{\sigma}(\varphi) \leqslant \eta_{\sigma}\|\varphi\|_{h}^{2} \quad \text { with } \quad \eta_{\sigma}=M_{\sigma} \alpha_{\sigma}^{2}, \\
\alpha_{\sigma}=1+h L e^{\sigma h}, \quad M_{\sigma}=\left\|U_{\sigma}(0)\right\|,
\end{gathered}
$$

where $U_{\sigma}(\tau)$ is the Lyapunov matrix corresponding to system (6). Similarly, according to Theorem 1 ,

$$
v(\varphi) \geqslant \mu\|\varphi(0)\|^{2}, \varphi \in S,
$$

where $\mu=\lambda_{\min }(W) \delta / 4$, and $\delta>0$ is the solution of the equation

$$
\alpha K e^{K \delta}=\frac{1}{2 \delta},
$$

here $K=\left\|A_{0}\right\|+\left\|A_{1}\right\|+g h$. Hence,

$$
v_{\sigma}(\varphi) \geqslant \mu_{\sigma}\|\varphi(0)\|^{2}, \varphi \in S,
$$

where $\mu_{\sigma}=\lambda_{\min }(W) \delta_{\sigma} / 4$, and $\delta_{\sigma}>0$ is the solution of the equation

$$
\alpha_{\sigma} K_{\sigma} e^{K_{\sigma} \delta_{\sigma}}=\frac{1}{2 \delta_{\sigma}}, \quad \text { where } \quad K_{\sigma}=\left\|A_{0}+\sigma I\right\|+e^{\sigma h} L .
$$

Consider first the functional $v_{\sigma}(\varphi)$, and choose $t^{*}$ such that $y_{t^{*}} \in S$. Then for the solution $y(t, \varphi)$ of system (6) we can write

$$
\|y(t, \varphi)\|^{2} \leqslant \frac{1}{\mu_{\sigma}} v_{\sigma}\left(y_{t^{*}}(\varphi)\right) \leqslant \frac{1}{\mu_{\sigma}} v_{\sigma}(\varphi) \leqslant \frac{\eta_{\sigma}}{\mu_{\sigma}}\|\varphi\|_{h}^{2} .
$$

If we use the functional $v(\varphi)$, then we obtain

$$
\begin{gathered}
\|y(t, \varphi)\|^{2} \leqslant \frac{1}{\mu} v\left(y_{t^{*}}(\varphi)\right) \leqslant \frac{1}{\mu}\left(v(\varphi)+\chi_{\sigma} \int_{-h}^{0}\|\varphi(s)\|^{2} d s\right) \\
\leqslant \frac{\beta_{\sigma}}{\mu}\|\varphi\|_{h}^{2}, \text { where } \chi_{\sigma}=M L\left(\sigma h+(\alpha+h L)\left(e^{\sigma h}-1\right)\right), \\
\beta_{\sigma}=\eta+\chi_{\sigma} h=M\left(\alpha^{2}+\sigma L h^{2}+h L(\alpha+h L)\left(e^{\sigma h}-1\right)\right),
\end{gathered}
$$

here $\sigma$ is supposed to satisfy (7). For system (1), the estimates of this kind lead to the exponential estimates of solutions:
Theorem 4. If system (1) is exponentially stable and $\sigma$ satisfies inequality (7), then for every solution of system (1) the following estimates hold

$$
\begin{gathered}
\|x(t, \varphi)\| \leqslant \gamma_{i}(\sigma) e^{-\sigma t}\|\varphi\|_{h}, \quad t \geqslant 0, i=1,2, \text { where } \\
\gamma_{1}(\sigma)=\sqrt{\frac{\eta_{\sigma}}{\mu_{\sigma}}} \text { and } \gamma_{2}(\sigma)=\sqrt{\frac{\beta_{\sigma}}{\mu}} .
\end{gathered}
$$

The examples show that in some cases our robust stability condition (Theorem 2) and estimation of $\sigma$ (Theorem 3) give better results than the results obtained with the help of the complete-type functionals, see [7] or [12].

\section{CONClusion}

In the paper, the robust stability condition (Theorem 2) and the exponential estimates for solutions (Theorem 4) are presented for a linear differential system with distributed delay.

\section{ACKNOWLEDGMENT}

The author acknowledges Saint Petersburg State University for the research grant 9.37.157.2014.

\section{REFERENCES}

[1] R. Bellman and K. L. Cooke, Differential-Difference Equations. New York: Academic Press, 1963.

[2] C. Cuvas and S. Mondie, "Necessary conditions for the exponential stability of a class of distributed delay-systems," in Proc. IEEE 53rd Annu. Conf. Decision \& Control, Los Angeles, CA, USA, 2014, pp. 1960-1965.

[3] A. V. Egorov and S. Mondie, "The delay Lyapunov matrix in robust stability analysis of time-delay systems," in Proc. 12th IFAC Workshop Time-Delay Systems (TDS'15), Ann Arbor, MI, USA, Jun. 2015, pp. 245250.

[4] W. Huang, "Generalization of Liapunov's theorem in a linear delay system,” J. Math. Anal. and Applicat., vol. 142, pp. 83-94, 1989.

[5] N. N. Krasovskii, "On the second Lyapunov method application to the equations with delay," Prikl. Mat. Mekh., (in Russian), vol. 20, pp. 315327, 1956.

[6] V. L. Kharitonov, "Lyapunov matrices for a class of time-delay systems," Systems \& Control Letters, vol. 55, pp. 610-617, 2006.

[7] V. L. Kharitonov, Time-Delay Systems: Lyapunov Functionals and Matrices. Basel: Birkhäuser, 2013.

[8] V. L. Kharitonov and D. Hinrichsen, "Exponential estimates for timedelay systems," Systems \& Control Letters, vol. 53, pp. 395-405, 2004.

[9] I. V. Medvedeva and A. P. Zhabko, "Synthesis of Razumikhin and Lyapunov-Krasovskii approaches to stability analysis of time-delay systems," Automatica, vol. 51, pp. 372-377, Jan. 2015.

[10] I. V. Medvedeva and A. P. Zhabko, "A novel approach to robust stability analysis of linear time-delay systems," in Proc. 12th IFAC Workshop on Time-Delay Systems (TDS'15), Ann Arbor, MI, USA, Jun. 2015, pp. 233238.

[11] Y. M. Repin, "Quadratic Lyapunov functionals for systems with delay," J. Appl. Math. Mech., vol. 29, pp. 669-672, 1965 (Transl.: Prikl. Mat. Mekh., 29, 1965, pp. 564-566).

[12] O. Santos, V. L. Kharitonov and S. Mondie, "Quadratic functional for systems with distributed time delays," in Proc. 16th IFAC World Congr., Prague, Czech Republic, 2005, pp. 454-459.

[13] O. Santos, S. Mondie and V. L. Kharitonov, "Robust stability conditions for systems with distributed delays," in Proc. 45th IEEE Conf. Decision \& Control, San Diego, CA, USA, 2006, pp. 217-222. 